\title{
On Optimization of Predictions in Ontology-Driven Situation Awareness
}

\author{
Norbert Baumgartner ${ }^{1}$, Wolfgang Gottesheim ${ }^{2}$, Stefan Mitsch ${ }^{2}$, \\ Werner Retschitzegger ${ }^{3}$, and Wieland Schwinger ${ }^{2}$ \\ 1 team Communication Technology Mgt. Ltd., Goethegasse 3, 1010 Vienna, Austria \\ 2 Johannes Kepler University Linz, Altenbergerstr. 69, 4040 Linz, Austria \\ 3 University of Vienna, Dr.-Karl-Lueger-Ring 1, 1010 Vienna, Austria
}

\begin{abstract}
Systems supporting situation awareness in large-scale control systems, such as, e.g., encountered in the domain of road traffic management, pursue the vision of allowing human operators prevent critical situations. Recently, approaches have been proposed, which express situations, their constituting objects, and the relations in-between (e.g., road works causing a traffic jam), by means of domain-independent ontologies, allowing automatic prediction of future situations on basis of relation derivation. The resulting vast search space, however, could lead to unacceptable runtime performance and limited expressiveness of predictions. In this paper, we argue that both issues can be remedied by taking inherent characteristics of objects into account. For this, an ontology is proposed together with optimization rules, allowing to exploit such characteristics for optimizing predictions. A case study in the domain of road traffic management reveals that search space can be substantially reduced for many real-world situation evolutions, and thereby demonstrates the applicability of our approach.
\end{abstract}

\section{Introduction}

Situation awareness. Situation awareness is gaining more and more importance as a way to help human operators cope with information overload in large-scale control systems, as, e.g., encountered in the domain of road traffic management. In this respect, a major vision of situation-aware systems is to support human operators in anticipating possible future situations in order to pro-actively prevent critical situations by taking appropriate actions. As first steps towards making this vision come true, situation-aware systems aggregate information about physical objects (e.g., road works) and relations among them (e.g., causes) into relevant situations (e. g., road works cause a traffic jam).

Situation evolution and prediction. Situations evolve continuously, resulting from alterations of real-world objects over time (e.g., road works cause a traffic jam which grows, then shrinks, and finally dissolves). Such evolutions are

\footnotetext{
* This work has been funded by the Austrian Federal Ministry of Transport, Innovation and Technology (BMVIT) under grant FIT-IT 819577.
} 
also dealt with in the fields of context prediction [1] and time series analysis [2] to predict future developments. However, techniques proposed in these fields are often based on quantitative data and make use of learning from historic data to achieve domain-independence. Therefore, they are only able to detect situations that have already occurred in the past, and, hence, are not applicable for predicting critical situations before they occur for the first time [1]. In situation awareness, however, such critical situations often endanger life and are, besides that, not observable in sufficient quantity to obtain meaningful training data for machine learning (e.g., a wrong-way driver rushing into a traffic jam). In our previous work [3],[4],[5],[6], we therefore proposed - in accordance with Llinas et al. [7] — to pursue a different approach using domain-independent ontologies describing qualitative facts for achieving situation awareness, as well as techniques thereupon predicting future situations without relying on historic data. For this, we described situation evolution in terms of transitions of the relations contributing to a situation. Based on this, prediction of whether or not a current situation can evolve into a critical situation, is achieved by computing all possible paths of such transitions between the current and the critical situation. Optimization potentials for prediction. The number of such paths, however, depends on the contributing objects and the relations defined among them, resulting in a vast search space of possible transitions between two situations [5]. This holds the risk of inducing unacceptable runtime performance and limiting the expressiveness of predictions. We argue that both issues can be remedied by taking into account inherent characteristics of involved objects, describing in detail which relations among them are actually possible, and which transitions between these relations may occur. Up to now, such knowledge is often incorporated only implicitly, like, e. g., in terms of subsumption rules for assessing situations [8]. In this paper, we propose a domain-independent ontology for modeling object evolution characteristics describing how a real-world object anchored in time and space can change, and based on this, optimization rules for reducing prediction search space.

Structure of the paper. In Section 2, we summarize our previous work on situation awareness and exemplify the potentials of our approach in the domain of road traffic management. In Section 3, an ontology together with optimization rules is proposed for representing and exploiting object evolution characteristics. Next, we evaluate the applicability of such characteristics on basis of a case study in Section 4. Finally, we provide an overview of related work in Section 5, before we conclude the paper in Section 6 by indicating further prospects of our work.

\section{Motivating Example}

Road traffic management systems, responsible for, e.g., ensuring safe driving conditions, are a typical application domain for situation awareness. In this section, examples from the domain of road traffic management are used to summarize our previous work on situation awareness and to illustrate the potentials of incorporating object evolutions characteristics into prediction of situations. 
Situation awareness in road traffic management. In principle, human operators of road traffic management systems observe highways for critical situations like, e.g., a wrong-way driver heading towards a traffic jam, in order to resolve them by taking appropriate actions. In our previous work [4] we introduced a framework for building situation-aware systems on basis of a domainindependent ontology. This framework was used to build a prototype [6] supporting human operators and, thereby, the feasibility of our approach to situation awareness was shown. In the framework's ontology, objects and relations among them, which are derived from object properties, are aggregated into situations. Information on such objects and relations (and thereby on traffic situations) is obtained from various sources such as, e. g., from traffic flow monitoring systems and drivers reporting traffic information. Changes of object properties over time, like, e. g., movement on a road, reported by such sources cause changes in the relations between objects, leading in turn to situation evolution.

Spatial and temporal relations. For describing such relations between objects, we discern families of spatial and temporal relations, with each family modeling a certain real-world aspect [3]. These comprise mereotopological reasoning about regions, describing, e.g., whether a traffic jam occurs in a tunnel, positional and orientational reasoning about points, expressing, e. g., that an accident happened in front of a traffic jam, as well as size and distance. Temporal relation families allow us to express that, e. g., an accident occurred shortly before a traffic jam. For describing these relation families, we base upon well-known calculi further detailed in Section 3.

Predicting future situations. By employing these relation calculi, prediction of situations provides the basis for early detection of possibly emerging critical situations. Let us suppose that human operators of a road traffic management system want to be informed of a critical situation "Wrong-way driver in the area of road works", as depicted in Fig. 1. In order to pro-actively take actions such as, e. g., issue warnings to motorists, human operators want to be informed already if such a situation is possibly emerging, which is indicated by the initial situation "Wrong-way driver very close to road works". In Fig. 1, we illustrate the combination of relations from appropriate relation families - in this case, mereotopology, distance, orientation, and size - which have to be valid between objects in order to constitute the initial situation. All other situations in this example would be formalized with similar combinations, but details are left out for brevity. For predicting whether or not this situation can evolve into the critical situation, we derive all possible paths of transitions between these two situations (Fig. 1 shows an exemplary subset thereof). A transition between two such situations occurs, if a transition is possible in at least one of the contributing relation families. By that, we can predict to reach the situation depicted in step 1 with one transition describing a change in the distance between the wrong-way driver and road works. With one further step - one transition in our relation family describing mereotopology and one transition in the family describing spatial distance - we predict to reach the critical situation. Due to relation families being defined independently from concrete objects, alternative paths via different 


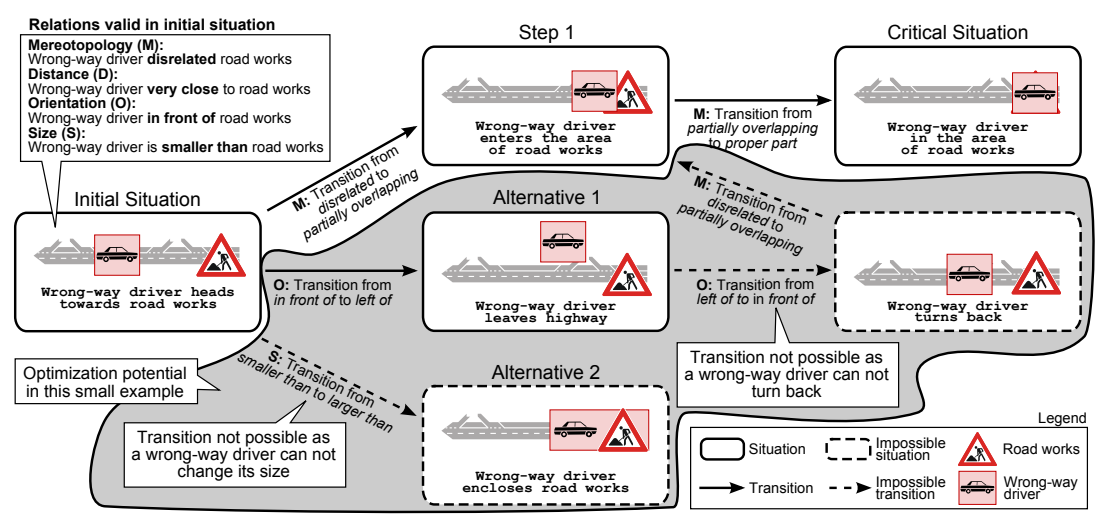

Fig. 1. Exemplary prediction of situations towards a critical situation.

situations are predicted, as depicted in Fig. 1. However, not all of these predicted situations and paths can actually occur in real-world.

Considering object evolution. The approach we present in this paper aims to eliminate such impossible future situations and transitions between them by incorporating object evolution characteristics into the prediction process. Prerequisite for this is to remove impossible relations and transitions from relation families. For example, knowledge that a wrong-way driver can only change its position, but obviously not its size, allows us to rule out a predicted future situation, in which the wrong-way driver encloses the area of road works, as depicted in Fig. 1. Another impossible situation occurs, if our information sources report that the wrong-way driver turned left at the depicted junction (i. e., leaving the highway), instead of following step 1. Object evolution characteristics enable us to conclude that no further evolution towards the critical situation is possible, as road works can not change their position, and a wrong-way driver can not turn back towards the road works. Hence, in Fig. 1 we can exclude the situation "Wrong-way driver turns back" from the possible paths towards the critical situation, which allows us to take back issued warnings. Without object evolution characteristics, we would not be able to detect this circumstance, and would still predict that the critical situation is about to emerge.

Summarizing, by using object evolution characteristics, we are able to reduce the number of relevant relations and transitions in a relation family as a pre-requisite for removing impossible situations and transitions between them. Thereby, the number of possible paths between two situations, i.e., prediction search space, is reduced.

\section{Object Evolution Characteristics}

Object evolution characteristics, as laid out in the previous section, bear potential for increasing performance during prediction of future situations, as well as 
for increasing prediction expressiveness. In Fig. 2, the principle idea of our approach is depicted, by showing the interaction between the different constituents. An evolution ontology defines the vocabulary for describing object evolution characteristics, which is exploited by optimization rules to optimize relation families. On basis of such optimized relation families, a prediction algorithmpresented in our previous work [5] - generates optimized possible evolutions between situations. In the following, these constituents are described in detail.

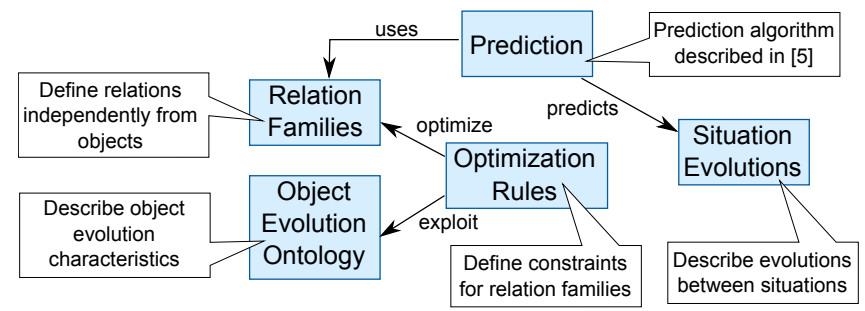

Fig. 2. Overview of our approach.

Object evolution ontology. Situation evolution, as described above, depends on the ability of objects to change over time. Considering object evolution characteristics in more detail, one can naturally differentiate between situationindependent ones describing inherent characteristics of objects that do not affect or depend on other objects (e.g., road works can not change their size), and situation-dependent ones defining how an object evolves in relation with other objects (e. g., a traffic jam caused by an accident in front of it can only dissolve after the accident has been cleared). Although the potential benefit of situationdependent object evolution characteristics are likely to be higher than those of situation-independent ones, they incur a fundamental increase in modeling complexity, as object evolution characteristics would have to be modeled individually for every situation. Therefore, in the object evolution ontology proposed in the following, we focus on situation-independent object evolution characteristics, and later show in Section 4 that, thereby, already substantial optimizations can be achieved with reasonable modeling effort.

We differentiate in the ontology depicted in Fig. 3 between non-changing objects and changing objects being able to change at least in some of their properties, thus contributing to situation evolution. As objects in situation awareness are anchored in time and space, corresponding types describe an object's typical lifespan and size, as well as an object's particular kind of supported spatial change (scaling - changes size, translational motion - changes position, and rotation $^{1}$ —changes orientation). A complementary characteristic - boundary permeability-describes, whether or not objects are able to share the same region

${ }^{1}$ In the field of computer graphics, a fourth change - reflection-is known, but this is of little practical benefit for describing real-world objects in situation awareness. 


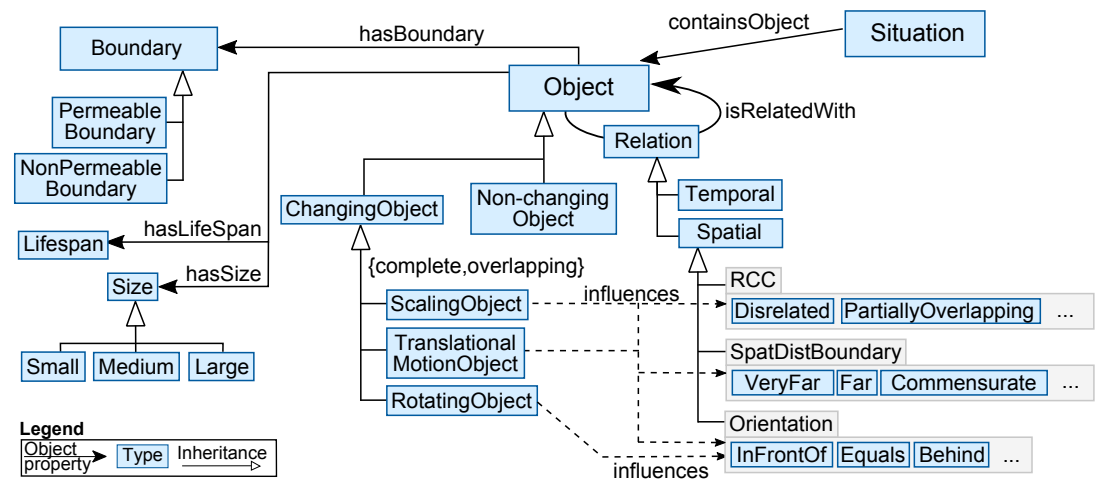

Fig. 3. Ontology for describing object evolution characteristics.

with other objects (e.g., a tunnel has a permeable boundary, meaning that other objects can be inside the tunnel). The object evolution characteristics of concrete objects (e.g., a wrong-way driver) are then modeled by inheriting from the appropriate types of the evolution ontology (cf. Table 2 for an exemplary tabular representation).

Relation families. For formalizing the relation families introduced in Section 2, we use well-known calculi from the field of spatio-temporal reasoning: the Region Connection Calculus (RCC, cf. [9]) describes mereotopology, Spatial Distance of Boundaries (SpatDistBoundary, cf. [10]) formalizes spatial distance, and the Oriented Points Relation Algebra (OPRA, cf. [11]) describes orientation. Each relation family is described with a directed graph representing relations between objects as nodes and possible transitions in-between as edges (called conceptual neighborhood graph - CNG [12]), as depicted in Figure $4^{2}$.

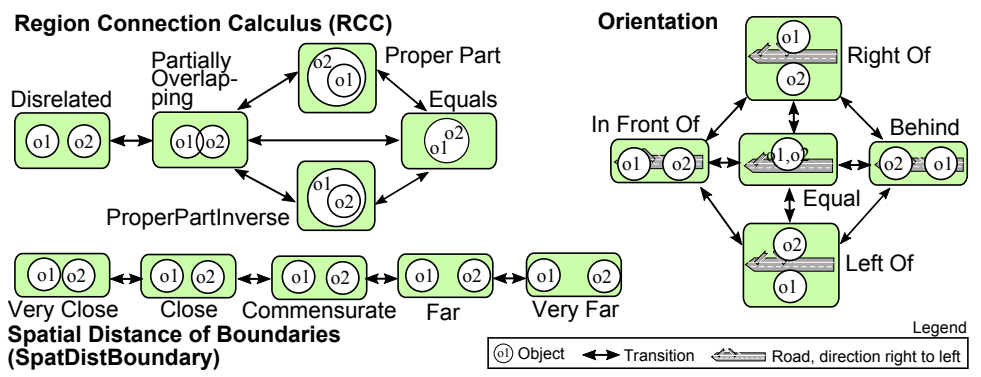

Fig. 4. Conceptual neighborhood graphs of relation families.

\footnotetext{
${ }^{2}$ Note that in this paper we use a rather basic notion of an orientation calculus describing relative orientation between two objects on their centroids in order to increase comprehensibility. In contrast, OPRA would result in a very large CNG.
} 
Optimization rules. On basis of the ontology introduced above, optimizations on the level of single relation families are enabled using optimization rules detailed in the following. Relation families describing mereotopology, distance, and orientation are influenced by types representing object evolution characteristics, and thereby drive the formalization of optimization rules. In particular, mereotopological relations are influenced by characteristics describing whether and how objects support translational motion or scaling, and whether an object's boundaries are permeable. For example, a car having a non-permeable boundary can be a proper part of a tunnel having a permeable boundary, but the inverse relation is not possible. Relations describing positions and distances are influenced by translational motion and scaling only. For example, road works and accidents can not move, inducing that the spatial distance between them will not change throughout their lifespan. Finally, relations describing orientation are influenced by rotation, translational motion, and scaling.

Optimization rules - on basis of the object evolution characteristics - define conditions for removing relations and transitions from the relation family's CNG. These optimization rules are formalized in terms of a simplified syntax based on the Semantic Web Rule Language (SWRL, [13]). In this syntax, an object o being a member of the type $\mathrm{T}$ is expressed as $\mathrm{T}(\mathrm{o})$, whereas two objects 01 and 02 taking part in the relation $\mathrm{R}$ are expressed as $\mathrm{R}(01,02)$. Table 1 lists optimization rules for the relations and transitions of the three relation families RCC, SpatDistBoundary, and Orientation together with their effect on relations and transitions. In general, it can be seen that, concerning RCC, optimization rules reduce the number of possible relations and transitions. We pick out the

Table 1. Optimization rules for three relation families.

\begin{tabular}{|c|c|c|}
\hline & Optimization Rule & Optimization Effect \\
\hline \multicolumn{3}{|c|}{$\mathrm{RCC}$} \\
\hline $\begin{array}{l}1 \\
2 \\
3 \\
4\end{array}$ & 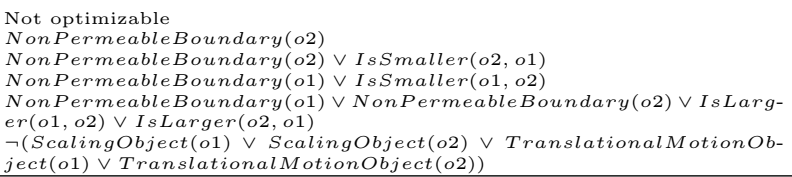 & $\begin{array}{l}\text { Disrelated is not removable } \\
\text { Removes Partially0verlapping (o1, 02) } \\
\text { Removes ProperPart }(01,02) \\
\text { Removes ProperPartInverse }(01,02)) \\
\text { Removes Equals }(01,02) \\
\text { Removes all transitions }\end{array}$ \\
\hline \multicolumn{3}{|c|}{ SpatDistBoundary } \\
\hline 6 & $\begin{array}{l}\text { Not optimizable } \\
\neg(\text { ScalingObject }(o 1) \vee \text { ScalingObject }(o 2) \vee \text { TranslationalMotionOb- } \\
\text { ject }(o 1) \vee \text { TranslationalMotionObject }(o 2))\end{array}$ & $\begin{array}{l}\text { Relations are not removable } \\
\text { Removes all transitions }\end{array}$ \\
\hline \multicolumn{3}{|c|}{ Orientation } \\
\hline $\begin{array}{l}7 \\
8\end{array}$ & $\begin{array}{l}\text { Not optimizable } \\
\neg(\text { ScalingObject }(\text { o1 }) \vee \text { ScalingObject }(o 2) \vee \text { TranslationalMotionOb- } \\
\text { ject }(\text { o1 }) \vee \text { TranslationalMotionObject }(\text { o } 2)) \\
\neg((\text { RotatingObject }(\text { o } 1) \wedge(\text { ScalingObject }(o 1) \vee T \text { ranslationalMotionOb- } \\
\text { ject }(\text { o } 1)) \vee \vee(\text { RotatingObject }(o 2) \wedge(\text { ScalingObject }(o 2) \vee T r a n s l a t i o n a l- \\
\text { MotionObject }(\text { o } 2))))\end{array}$ & $\begin{array}{l}\text { Relations are not removable } \\
\text { Removes InFrontof } \leftrightarrow \text { Equal and re- } \\
\text { moves Equal } \leftrightarrow \text { Behind } \\
\text { Removes all other transitions }\end{array}$ \\
\hline
\end{tabular}

relation ProperPart of RCC to exemplify the meaning of these optimization rules: ProperPart $(01,02)$ between the objects 01 and 02 is removed from RCC and, therefore, not taken into account during prediction of situations, if o2 does not have a permeable boundary and if 02 is smaller than 01 . All other rules can 
be interpreted analogously. With similar rules on combined relations of different relation families, we are able to optimize complex situation types. The optimizations achievable for different object combinations and transitions between situations are shown in the next section.

\section{Road Traffic Management Case Study}

In this section, we employ a case study in the domain of road traffic management to discuss the potentials of situation-independent object evolution characteristics for optimizing prediction of future situations. For this, we base upon road traffic objects with their evolution characteristics, as well as situations defined in collaboration with the Austrian highways agency ASFINAG ${ }^{3}$ (cf. [14] for a detailed overview of the more than 100 road traffic objects, and 16 critical situations considered in our prototype). In Table 2, we use the evolution ontology introduced above to describe evolution characteristics of a small subset of these objects, which was selected to show each spatial characteristic at least once.

Table 2. Evolution characteristics of real-world objects.

\begin{tabular}{llllll}
\hline Object & Size & $\begin{array}{l}\text { Translational- } \\
\text { MotionObject }\end{array}$ & $\begin{array}{l}\text { Scaling- } \\
\text { Object }\end{array}$ & $\begin{array}{l}\text { Rotating- } \\
\text { Object }\end{array}$ & Boundary \\
\hline RoadWorks & Large & - & - & - & PermeableBoundary \\
Accident & Medium & - & - & - & NonpermeableBoundary \\
WrongWayDriver & Small & $\checkmark$ & - & $\checkmark$ & NonpermeableBoundary \\
TrafficJam & Large & $\checkmark$ & $\checkmark$ & $\checkmark$ & PermeableBoundary
\end{tabular}

Optimization of a single relation family's relations and transitions. To start with, we evaluate the effects of these object evolution characteristics on relations and transitions of single relation families. Fig. 5 shows the optimizations for the RCC relation family achieved by applying the optimization rules introduced above to the objects Accident and RoadWorks described in Table 2. Naturally, these objects with few evolution characteristics are best suited

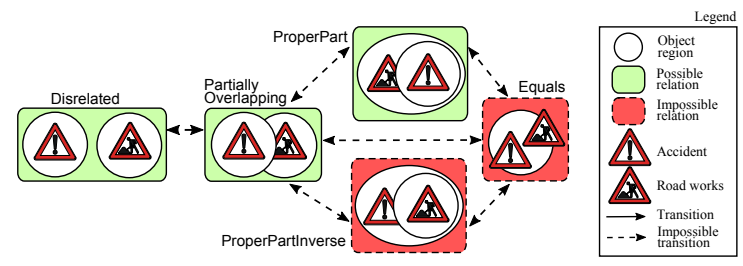

Fig. 5. Optimized RCC relation family.

to reduce the CNGs of relation families. For example, as neither road works

\footnotetext{
${ }^{3}$ www.asfinag.at
} 
nor accidents can change their position or size, optimization rule 5 removes all transitions from RCC. This means that, once a particular relation between two objects is detected, no further evolution can occur. Additionally, the relations ProperPartInverse and Equals are removed by the optimization rules 3 and 4, because an accident's non-permeable boundary does not allow other objects to enter the region occupied by the accident. These optimizations reduce the search space, because less relations and/or transitions need to be taken into account during prediction of situations. At the same time, prediction expressiveness is increased, because only those relations and transitions being actually relevant for the involved objects are used for predicting situations. For example, on basis of such an optimized CNG, as depicted in Fig. 5, we no longer would predict that an accident can become ProperPartInverse of road works. Similar optimizations are also possible between wrong-way drivers and traffic jams. If objects are capable to change in many ways, like, e.g., traffic jams do, such optimizations are, as already mentioned, not possible.

Optimization of situations and transitions between two situations. As described before, relation families optimized in such a way reduce the number of situations and transitions towards a critical situation. In Fig. 6, a wrong-way driver is close to a traffic jam, indicating a possibly emerging critical situation, which is reached when the wrong-way driver rushes into the traffic jam. In general, intermediary situations and transitions between these two situations are predicted if, as mentioned above, a transition is possible in at least one of the contributing relation families. Without taking object evolution characteristics

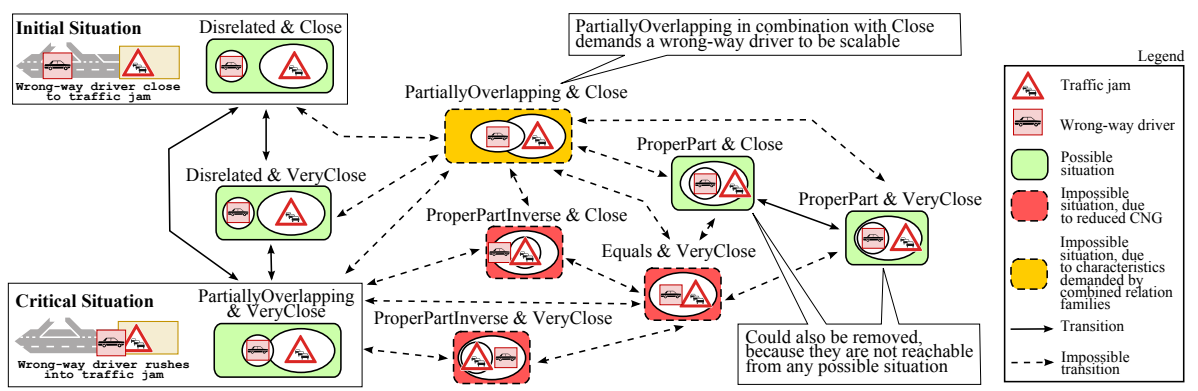

Fig. 6. Situation evolution graph with optimized transitions.

into account, prediction would result in a large number of such intermediary situations and transitions. From these, first of all three situations with their corresponding transitions can be ruled out employing the optimized CNGs, because they rely on impossible relations. Additionally, another situation is not possible, because its combination of relations demands object evolution characteristics not being supported by the wrong-way driver: the situation PartiallyOverlapping \& Close describes that a wrong-way driver and a traffic jam partially overlap (i. e., a wrong-way driver is partly outside and partly inside a traffic jam), while 
at the same time their boundaries are close to each other. This is not possible, because the size of a wrong-way driver only allows VeryClose when overlapping another object. Such an optimization rule is not listed in Table 1, because it is actually situation-dependent. Overall, the optimizations reduce the number of possible situations between the initial and the critical situation from nine to five, and the transitions from 19 to four. If additionally removing non-reachable situations, a further reduction to three situations, and three transitions is possible. Optimization on basis of semantics of combined relation families. However, in this example we still find transitions, which are actually not possible in a real-world scenario. These impossible transitions are characterized by implicit semantics resulting from combined relations. For example, we predict to reach PartiallyOverlapping \& VeryClose with a single transition from Disrelated \& Close, because it only requires one transition in RCC and one transition in SpatDistBoundary. But two real-world objects would first become Disrelated \& VeryClose, before they can become PartiallyOverlapping. Removing such transitions will be subject of our ongoing work (cf. Section 6).

Summary of optimization results. In Table 3, we summarize the achievable optimizations for ten out of the 16 situations defined with ASFINAG. For formally describing the situations, we use the syntax introduced for our optimization rules in Section 3. We then compare the number of situations and transitions predicted between an initial and a critical situation with and without considering object evolution characteristics. In general, it can be seen that considering object evolution characteristics - unless the evolution paths are already optimal, as it is the case in the fourth example - leads to a substantial reduction of both situations and transitions between them by more than $50 \%$. Particularly interesting is the last example, which - caused by the initial situation not containing any relation - leads to a large number of possible situations and transitions, because potentially every situation comprising a combination of relations from the families RCC and Orientation leads to the critical situation. In such settings, object evolution characteristics are particularly helpful to reduce the search space: if taking into account that road works are stationary and that a traffic jam can only be caused by road works being in front of it, all situations not containing InFrontOf are invalid.

\section{Related Work}

In this section, we present related research on prediction techniques in various domains, as well as more closely related work on the optimization and combination of calculi stemming from the area of qualitative spatial reasoning.

Prediction in other domains. Predicting future situations based on current and historic data is a task relevant in numerous domains. Various techniques in the field of time-series analysis, often being based on quantitative data, exist to, e.g., forecast future developments of stocks [2]. Efforts applying these approaches to the domain of road traffic management exist [15], but naturally they do not focus on predicting situations, but instead aim to predict traffic flow 
Table 3. Summary of achievable optimizations.

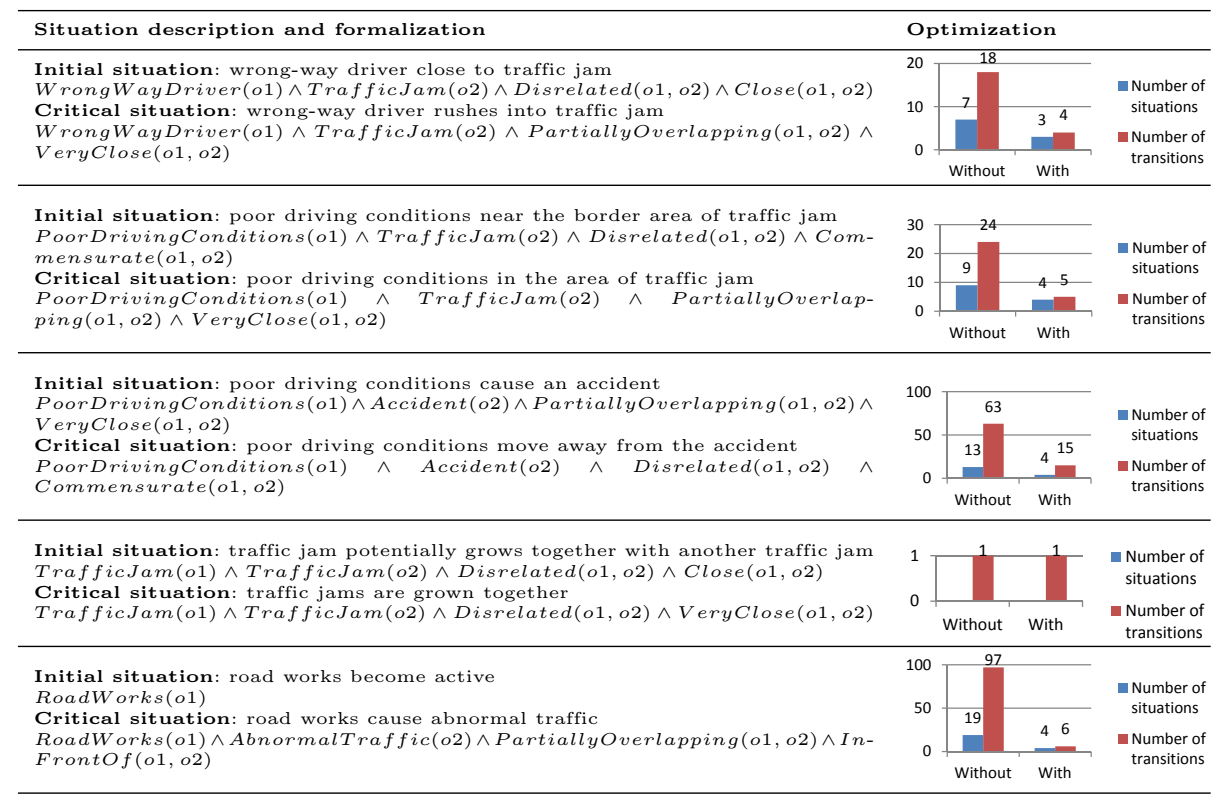

in terms of vehicle throughput. Solutions relying on alternative techniques, but still being based on quantitative data, like, e. g., neural networks [16] or bayesian belief networks [17], in a similar manner only predict traffic flow. In [18], a neural network-based solution to predict traffic accidents based on recognizing patterns in vehicle tracking data is presented. Compared to our work, this approach is tailored to the specific problem of accident prediction, whereas we aim for prediction of arbitrary situations from qualitative data in a domain-independent manner. Research in the area of context awareness postulates object evolution characteristics [19] and proposes to predict a situation's future on its historic attribute values. However, similar to the work on context history presented in [1], such an approach is unable to predict events that did not occur in the past.

Optimization of relation calculi. Dylla et al. [20] analyze a single calculus (OPRA) for optimization by manually eliminating transitions between relations being impossible due to characteristics like, e. g., locomotion. In contrast to this work, we propose domain-independent concepts for describing such characteristics and apply these concepts to automatically optimize different calculi. Moreover, we describe optimizations for combined calculi, which are frequently used in situation awareness to define situations. Possible combination methods, as well as comparisons of their reasoning performance are presented in Wölfl et al. [21]. The authors differentiate between orthogonal combinations expressing new calculi by combining existing formalisms without semantic interdependencies, and non-orthogonal combinations consisting of calculi with semantic interdependencies. In situation awareness, we typically encounter non-orthogonal combina- 
tions, which should best be combined using so-called tight integration techniques, as Wölfl et al. suggest. Such tight integrations are more expressive -i. e., contain more relations - than loose ones, which might due to their algorithmic design already rule out important relations. However, this advantage has its downside in prediction performance, as a large number of relations induces a large number of predictable situations and transitions. With our approach, we argue that prediction performance of tightly integrated calculi can be improved by removing impossible relations not fitting the characteristics of involved objects.

\section{Future Work}

We aim for further extending our object evolution ontology with additional object evolution characteristics, and in particular, with types for describing object evolution patterns. Such patterns promise a finer-grained representation of an object's changes over time (e.g., a traffic jam first grows, then begins to shrink, and finally dissolves), facilitating prediction of future situations with knowledge about complex object behavior. By recognizing these patterns we envision to provide operators of systems supporting situation awareness with better recommendations for actions to be taken. Second, our optimization rules are to be extended with situation-dependent rules, and with rules exploiting semantics of combined relation families, which are currently only implicitly available in ontology-driven situation awareness. Finally, we want to measure the effects on runtime performance resulting from removing relations and transitions of single relation families, as well as from removing situations and transitions between them from predicted situation evolutions. For this, we will extend our prototype and base on real-world test data provided by ASFINAG.

\section{References}

1. Mayrhofer, R., Radi, H., Ferscha, A.: Recognizing and predicting context by learning from user behavior. Radiomatics: Journal of Communication Engineering, special issue on Advances in Mobile Multimedia 1(1) (2004) 30-42

2. Cheng, C.H., Teoh, H.J., Chen, T.L.: Forecasting stock price index using fuzzy time-series based on rough set. 4th Intl. Conf. on Fuzzy Systems and Knowledge Discovery 3 (2007) 336-340

3. Baumgartner, N., Retschitzegger, W.: Towards a situation awareness framework based on primitive relations. In: Proc. of the IEEE Conf. on Information, Decision, and Control, Adelaide, Australia, IEEE (2007) 291-295

4. Baumgartner, N., Retschitzegger, W., Schwinger, W.: Lost in time, space, and meaning - an ontology-based approach to road traffic situation awareness. In: Proc. of the 3rd Workshop on Context Awareness for Proactive Systems, Guildford, UK (2007)

5. Baumgartner, N., Retschitzegger, W., Schwinger, W., Kotsis, G., Schwietering, C.: Of situations and their neighbors-Evolution and Similarity in Ontology-Based Approaches to Situation Awareness. In: Proc. of the 6th Intl. and Interdisciplinary Conf. on Modeling and Using Context, Roskilde, Denmark, Springer (2007) 29-42 
6. Baumgartner, N., Retschitzegger, W., Schwinger, W.: A software architecture for ontology-driven situation awareness. In: Proc. of the 23rd Annual ACM Symposium on Applied Computing, Fortaleza, Ceara, Brazil, ACM (2008) 2326-2330

7. Llinas, J., Bowman, C., Rogova, G., Steinberg, A.: Revisiting the JDL data fusion model II. In: Proc. of the 7th Intl. Conf. on Information Fusion, Stockholm, Sweden (2004) 1218-1230

8. Kokar, M.M., Matheusb, C.J., Baclawski, K.: Ontology-based situation awareness. International Journal of Information Fusion 10(1) (2009) 83-98

9. Cohn, A.G., Bennett, B., Gooday, J.M., Gotts, N.: RCC: A calculus for region based qualitative spatial reasoning. GeoInformatica 1 (1997) 275-316

10. Hernández, D., Clementini, E., Felice, P.D.: Qualitative distances. In: Proc. of the Intl. Conf. COSIT '95, Semmering, Austria, Springer (1995) 45-57

11. Moratz, R., Dylla, F., Frommberger, L.: A relative orientation algebra with adjustable granularity. In: Proc. of the Workshop on Agents in Real-Time and Dynamic Environments. (2005)

12. Freksa, C.: Temporal reasoning based on semi-intervals. Artificial Intelligence 54(1) (1992) 199-227

13. Horrocks, I., Patel-Schneider, P.F., Boley, H., Tabet, S., Grosof, B., Dean, M.: SWRL: A semantic web rule language combining OWL and RuleML. http:// www.w3.org/Submission/SWRL (2004)

14. Baumgartner, N.: BeAware! - An Ontology-Driven Framework for Situation Awareness Applications. PhD thesis, Johannes Kepler University Linz (2008)

15. Sabry, M., Abd-El-Latif, H., Yousef, S., Badra, N.: A time-series forecasting of average daily traffic volume. Australian Journal of Basic and Applied Sciences 1(4) (2007) 386-394

16. Dia, H.: An object-oriented neural network approach to short-term traffic forecasting. European Journal of Operational Research 131(2) (2001) 253 - 261

17. Sun, S., Zhang, C., Yu, G.: A bayesian network approach to traffic flow forecasting. IEEE Transactions on Intelligent Transportation Systems 7(1) (2006) 124-132

18. Hu, W., Xiao, X., Xiea, D., Tan, T.: Traffic accident prediction using vehicle tracking and trajectory analysis. In: Proc. of the 6th Intl. Conf. on Intelligent Transportation Systems. (2003)

19. Padovitz, A., Loke, S.W., Zaslavsky, A., Burg, B.: Towards a general approach for reasoning about context, situations and uncertainty in ubiquitous sensing: Putting geometrical intuitions to work. In: Proc. of the 2nd Intl. Symposium on Ubiquitous Computing Systems, Tokyo, Japan (2004)

20. Dylla, F., Wallgrün, J.O.: Qualitative spatial reasoning with conceptual neighborhoods for agent control. Journal of Intelligent Robotics Systems 48(1) (2007) $55-78$

21. Wölfl, S., Westphal, M.: On combinations of binary qualitative constraint calculi. In: Proc. of the 21st Intl. Joint Conference on Artificial Intelligence, Pasadena, CA, USA (2009) 967-972 